Pacific

Journal of

Mathematics

\title{
ON SCHWARZ-CHRISTOFFEL MAPPINGS
}

Martin Chuaqui And Christian Pommerenke 


\title{
ON SCHWARZ-CHRISTOFFEL MAPPINGS
}

\author{
Martin Chuaqui And Christian Pommerenke
}

\begin{abstract}
We extend previous work on Schwarz-Christoffel mappings, including the special cases when the image is a convex polygon or its complement. We center our analysis on the relationship between the pre-Schwarzian of such mappings and Blaschke products. For arbitrary Schwarz-Christoffel mappings, we resolve an open question from earlier work of Chuaqui, Duren and Osgood that relates the degrees of the associated Blaschke products with the number of convex and concave vertices of the polygon. In addition, we obtain a sharp sufficient condition in terms of the exterior angles for the injectivity of a mapping given by the Schwarz-Christoffel formula, and study the geometric interplay between the location of the zeros of the Blaschke products and the separation of the prevertices.
\end{abstract}

\section{Introduction}

The purpose of this paper is to provide further information about Schwarz-Christoffel mappings that adds to the results obtained in [Chuaqui et al. 2011; 2012]. We refer the reader to [Bhowmik et al. 2009] for related interesting work on concave functions.

Let $f$ be a Schwarz-Christoffel mapping of the unit disk $\mathbb{D}$ onto the interior of an $(n+1)$-gon. In other words, $f$ is a conformal map onto a domain in the extended complex plane whose boundary consists of finitely many line segments, rays or lines. In [Chuaqui et al. 2012], it is shown that the pre-Schwarzian of $f$ has the form

$$
\frac{f^{\prime \prime}}{f^{\prime}}=\frac{2 B_{1} / B_{2}}{1-z B_{1} / B_{2}}
$$

for some finite Blaschke products $B_{1}, B_{2}$ without common zeros, with respective degrees $d_{1}, d_{2}$ satisfying $d_{1}+d_{2}=n$. The polygon is convex if and only if $d_{2}=0$ (see also [Chuaqui et al. 2011]). The representation for $f^{\prime \prime} / f^{\prime}$ is obtained from a

Chuaqui was partially supported by Fondecyt Grant \#1110321.

MSC2000: primary 30C20, 30C35; secondary 30C45.

Keywords: Schwarz-Christoffel mapping, prevertices, convex, concave, univalent mapping, Blaschke product. 
well-known formula:

$$
\frac{f^{\prime \prime}}{f^{\prime}}=-2 \sum_{k=1}^{n+1} \frac{\beta_{k}}{z-z_{k}},
$$

where each $z_{k}$ is a prevertex and $2 \pi \beta_{k}$ is the exterior angle at $z_{k}$ (that is, $\pi$ minus the interior angle); we have $\sum_{k=1}^{n+1} \beta_{k}=1$. The formula (1-2) remains valid for polygons with one vertex at infinity. (The angle at infinity between two sides is, by definition, -1 times the angle determined, at their crossing in the plane, by the lines containing the sides.)

As a consequence of (1-1) and (1-2), the prevertices are shown to be the roots of the equation

$$
\frac{z B_{1}(z)}{B_{2}(z)}=1 .
$$

It is interesting that (1-3) corresponds to a polynomial equation of degree $n+1$ for which all roots are simple and lie on $|z|=1$. This is a particular feature of the pair of Blaschke products $B_{1}, B_{2}$ arising from Schwarz-Christoffel mappings. Note that the topological degree of $z B_{1} / B_{2}$ on $\partial \mathbb{D}$ is $1+d_{1}-d_{2}$, so that $z B_{1} / B_{2}$ must be traversing in the negative sense at many of the prevertices. In fact, as the proof of Theorem 2 shows, at a prevertex $z_{k}, z B_{1} / B_{2}$ is traversing $\partial \mathbb{D}$ in the positive or negative sense according to whether $f\left(z_{k}\right)$ is a convex or a concave vertex. It is also interesting to observe that when $d_{2}=0$, any solution of (1-1) will result in a univalent mapping because $1+\operatorname{Re}\left\{z f^{\prime \prime} / f^{\prime}\right\} \geq 0$. In this paper we answer the natural question of finding a geometric interpretation for the degree $d_{2}$, and show that this integer coincides with the number of concave vertices of the polygon.

In Section 2, we also address the case of Schwarz-Christoffel mappings $f$ onto the exterior of an $(n+2)$-gon, with the normalization $f(0)=\infty$. In [Chuaqui et al. 2012], we showed that the pre-Schwarzian of such a mapping is given by

$$
z \frac{f^{\prime \prime}}{f^{\prime}}=\frac{2}{z^{2}\left(B_{1} / B_{2}\right)-1},
$$

for finite Blaschke products $B_{1}, B_{2}$ without common zeros, with degrees $d_{1}, d_{2}$, respectively, for which $d_{1}+d_{2}=n$. The polygon is convex if and only if $d_{2}=0$ and, as before, we show in this paper that $d_{2}$ is equal to the number of concave vertices of the polygon.

Another issue we address in this paper is the question of when a solution of (1-2), or equivalently, of

$$
f^{\prime}(z)=\prod_{k=1}^{n+1}\left(z-z_{k}\right)^{-2 \beta_{k}}, \quad \sum_{k=1}^{n+1} \beta_{k}=1
$$


does indeed correspond to a univalent mapping. In Theorem 4 below we obtain the sharp sufficient condition $\sum_{k=1}^{n+1}\left|\beta_{k}\right| \leq 2$ for univalence. The result is optimal in the sense that there are nonunivalent solutions of (1-2) for which $\sum_{k=1}^{n+1}\left|\beta_{k}\right|$ differs from 2 by an arbitrarily small amount.

In Section 3 we obtain results on the separation of the prevertices of convex or concave Schwarz-Christoffel mappings, expressed in terms of the location of the zeros $a_{1}, \ldots, a_{n}$ of the Blaschke product $B_{1}$ that appears in (1-1) or (1-4) (recall that, in this case, $d_{2}=0$ ). The results are sharp, and show, for example, that the prevertices tend to be uniformly separated on $\partial \mathbb{D}$ when all $\left|a_{k}\right|$ are very small. Finally, in Section 4 we derive some necessary conditions for the location of the zeros of the Blaschke products $B_{1}, B_{2}$ in (1-1) and (1-4) for arbitrary polygonal mappings.

\section{Blaschke products and univalence}

In [Chuaqui et al. 2011] we revisit the classical theme of convex mappings. The starting point is the observation that such mappings correspond exactly to the solutions of

$$
\frac{f^{\prime \prime}}{f^{\prime}}=\frac{2 h}{1-z h},
$$

for some function $h$ analytic in $\mathbb{D}$ and bounded by 1 . The image $f(\mathbb{D})$ is the interior of a polygon if and only if $h$ is a finite Blaschke product. We can express $h$ in terms of $p=f^{\prime \prime} / f^{\prime}$ as

$$
h=\frac{p}{2+z p},
$$

and draw the following result.

Theorem 1. Let $h$ be analytic in $\mathbb{D}$ with $|h(z)| \leq 1$ everywhere. Then there exists a sequence $\left\{B_{n}\right\}_{n \in \mathbb{N}}$ of finite Blaschke products converging to h locally uniformly in $\mathbb{D}$.

Proof. Let $f$ be the convex mapping corresponding to $h$ as above, and let $\Omega_{n}$ be a sequence of convex polygons converging to $f(\mathbb{D})$ in the sense of Carathéodory. Properly normalized Schwarz-Christoffel mappings $f_{n}$ of $\mathbb{D}$ onto $\Omega_{n}$ will converge locally uniformly to $f$. Each mapping $f_{n}$ satisfies (1-1) for a certain finite Blaschke product $B_{1}=B_{1, n}$ and $B_{2}=1$. The theorem now follows by expressing $B_{1, n}$ in terms of the pre-Schwarzian of $f_{n}$.

Next, we give an answer to an important issue left unresolved in [Chuaqui et al. 2012], namely the connection between the degrees $d_{1}, d_{2}$ and the number of convex and concave vertices of the polygon.

Theorem 2. Let $f$ map $\mathbb{D}$ onto the interior of an $(n+1)$-gon, and let $B_{1}, B_{2}$ be the corresponding Blaschke products in the representation (1-1). Then $d_{2}$ is equal to the number of concave vertices, while $d_{1}+1$ is equal to the number of convex vertices. 
Proof. Let

$$
\varphi(t)=\arg \left\{e^{i t} \frac{B_{1}}{B_{2}}\left(e^{i t}\right)\right\},
$$

with a well-defined branch of the argument once its value has been assigned at one given vertex. In any case,

$$
\varphi^{\prime}(t)=1+e^{i t}\left(\frac{B_{1}^{\prime}}{B_{1}}\left(e^{i t}\right)-\frac{B_{2}^{\prime}}{B_{2}}\left(e^{i t}\right)\right)=1+\left|B_{1}^{\prime}\left(e^{i t}\right)\right|-\left|B_{2}^{\prime}\left(e^{i t}\right)\right| .
$$

On the other hand, we see from (1-1) and (1-2) that

hence

$$
\frac{B_{1} / B_{2}}{z B_{1} / B_{2}-1}=\sum_{k=1}^{n+1} \frac{\beta_{k}}{z-z_{k}}
$$

$$
\beta_{k}=\lim _{z \rightarrow z_{k}}\left(z-z_{k}\right) \frac{B_{1} / B_{2}}{z B_{1} / B_{2}-1}=\frac{B_{1} / B_{2}}{\left(z B_{1} / B_{2}\right)^{\prime}}\left(z_{k}\right)=\frac{1}{\varphi^{\prime}\left(t_{k}\right)},
$$

where we have written $z_{k}=e^{i t_{k}}$. We say that $z_{k}$ is convex or concave according to whether the polygon is convex or concave at $f\left(z_{k}\right)$. We conclude that $\varphi^{\prime}\left(t_{k}\right)$ is positive at convex prevertices and negative at concave prevertices. Furthermore, the points $z_{k}$ are the solutions of (1-3). Hence we see from (2-1) that, for all $k$,

$$
\varphi\left(t_{k}\right)=2 \pi j_{k}, j_{k} \in \mathbb{Z}, \quad \text { and } \quad \varphi(t) \neq 2 \pi j, t \neq t_{k} .
$$

It follows now that

$$
\int_{t_{k}}^{t_{k+1}} \varphi^{\prime}(t)= \begin{cases}2 \pi & \text { when } z_{k}, z_{k+1} \text { are both convex, } \\ 0 & \text { when } z_{k}, z_{k+1} \text { are one convex and one concave, } \\ -2 \pi & \text { when } z_{k}, z_{k+1} \text { are both concave. }\end{cases}
$$

Let $a$ be the number of consecutive convex prevertices, $b$ the number of instances a vertex of one type is followed by one of the other type, and $c$ the number of consecutive concave prevertices. Then $a+b+c=n+1$, and we see by (2-4) that

$$
\int_{0}^{2 \pi} \varphi^{\prime}(t) d t=2 \pi\left(1+d_{1}-d_{2}\right)=2 \pi(a-c) .
$$

Hence we have

$$
1+d_{1}+d_{2}=n+1=a+b+c, \quad 1+d_{1}-d_{2}=a-c .
$$

We conclude that

$$
1+d_{1}=a+\frac{b}{2}, \quad d_{2}=c+\frac{b}{2} .
$$

To obtain the theorem, we claim that $c+(b / 2)$ is equal to the number of concave vertices (or prevertices). To see this, let $z_{k}, \ldots, z_{l}$ be any maximal chain of consecutive concave prevertices. Hence $z_{k-1}$ and $z_{l+1}$ are convex prevertices. The 
collection $z_{k}, \ldots, z_{l}$ of concave prevertices contributes $l-k$ to the count of $c$ and contributes 2 to the count of $b$. Thus its contribution in the count of $c+(b / 2)$ is exactly the number of points in the chain. This proves the claim, and completes the proof of the theorem.

Similar results hold for mappings $f$ onto the exterior of an $(n+2)$-gon, having the important normalization $f(0)=\infty$. For such mappings we have that

$$
\frac{f^{\prime \prime}}{f^{\prime}}=2\left(\sum_{k=1}^{n+2} \frac{\beta_{k}}{z-z_{k}}-\frac{1}{z}\right),
$$

where, as before, $z_{k}$ are the prevertices and $2 \pi \beta_{k}$ are the exterior angles, which satisfy $-1<\beta_{k}<1$ and $\sum_{k=1}^{n+2} \beta_{k}=1$. In [Chuaqui et al. 2012], this was shown to lead to

$$
z \frac{f^{\prime \prime}}{f^{\prime}}=\frac{2}{z^{2}\left(B_{1} / B_{2}\right)-1}
$$

for Blaschke products $B_{1}, B_{2}$ of degree $d_{1}, d_{2}$ satisfying $d_{1}+d_{2}=n$. Again, the case $d_{2}=0$ corresponds exactly to when the polygon is convex. The prevertices appear as the solutions of the equation $z^{2} B_{1}=B_{2}$, yet no further information was provided in connection with the degrees of the Blaschke products. With a similar argument as in the proof of Theorem 2, one can show:

Theorem 3. Let $f$ map $\mathbb{D}$ onto the exterior of an $(n+2)$-gon, and let $B_{1}, B_{2}$ be the corresponding Blaschke products in the representation (1-4). Then $d_{2}$ is equal to the number of concave vertices, while $d_{1}+2$ is equal to the number of convex vertices.

Next, we address the question of the univalence of solutions of (1-2).

Theorem 4. Let $0 \leq t_{1}<\cdots<t_{n+1}<2 \pi, z_{k}=e^{i t_{k}}, \beta_{k} \in \mathbb{R}, k=1, \ldots, n+1$, and let

$$
\sum_{k=1}^{n+1} \beta_{k}=1, \quad \sum_{k=1}^{n+1}\left|\beta_{k}\right| \leq 2 .
$$

Then the function $f$ defined by

$$
f^{\prime}(z)=a \prod_{k=1}^{n+1}\left(z-z_{k}\right)^{-2 \beta_{k}}, \quad a \in \mathbb{C}, a \neq 0,
$$

is univalent in $\mathbb{D}$.

Observe that there exist polygons with $\sum\left|\beta_{k}\right|$ arbitrarily large for which $f$ remains univalent. For example, one can consider a polygon inscribed between two disjoint logarithmic spirals. On the other hand, once $\sum_{k=1}^{n+1}\left|\beta_{k}\right|$ is allowed to exceed 2 , then the sum of exterior angles at concave vertices will be larger than $\pi$ in absolute value, thus making it possible for the image $f(\mathbb{D})$ to intersect itself. 
Proof of Theorem 4. Let $f$ be given as in (2-6) and suppose that (2-5) holds. Then $f$ is locally injective in $\mathbb{D}$, and we will show that $f$ is univalent there, and, in fact, that it is close-to-convex. Among the various equivalent formulations of this geometric property (see, e.g., [Duren 1983, p. 48]), we will show for $0 \leq \theta_{1}<\theta_{2}<2 \pi$ that

$$
I=I\left(\theta_{1}, \theta_{2}\right)=\int_{\theta_{1}}^{\theta_{2}} \operatorname{Re}\left\{1+z \frac{f^{\prime \prime}}{f^{\prime}}(z)\right\} d \theta>-\pi, \quad z=r e^{i \theta} .
$$

To prove this, observe that

$$
\operatorname{Re}\left\{1+z \frac{f^{\prime \prime}}{f^{\prime}}(z)\right\}=\sum_{k} \beta_{k} \operatorname{Re}\left\{\frac{z_{k}+z}{z_{k}-z}\right\}=\sum_{k} \beta_{k} \frac{1-r^{2}}{\left|z_{k}-z\right|^{2}} .
$$

In trying to obtain a lower bound for $I$ we can discard the terms with $\beta_{k}>0$. For the other terms, we have that

$$
\int_{\theta_{1}}^{\theta_{2}} \frac{1-r^{2}}{\left|z_{k}-r e^{i \theta}\right|^{2}} d \theta<2 \pi
$$

because of the properties of the Poisson kernel. Hence

$$
I>2 \pi \sum_{\beta_{k}<0} \beta_{k} \geq-\pi,
$$

as desired.

Example. Avkhadiev and Wirths [2002; 2005; 2007] initiated the study of the so-called concave mappings, that is, univalent mappings of the disk $\mathbb{D}$ onto the complement of a convex set. As an example of Theorem 4 we can consider a convex polygon $P$ with $\infty \in P$ and the conformal mapping of $\mathbb{D}$ onto the complement of $P$. Let $\pi \lambda$ be the angle of $f(\mathbb{D})$ at $\infty$, with $1 \leq \lambda \leq 2$. It follows from [Avkhadiev and Wirths 2005] that

$$
f^{\prime}(z)=a\left(z-z_{n+1}\right)^{-\lambda-1} \prod_{k=1}^{n}\left(z-z_{k}\right)^{\gamma_{k}}, \quad \sum_{k=1}^{n} \gamma_{k}=\lambda-1,
$$

that is, $\beta_{k}=\frac{1}{2} \gamma_{k}$ for $k=1, \ldots, n$, and $\beta_{n+1}=\frac{1}{2}(1+\lambda)$. Therefore

$$
\sum_{k=1}^{n+1}\left|\beta_{k}\right|=\frac{1}{2}(\lambda+1)+\frac{1}{2}(\lambda-1)=\lambda \in[1,2] .
$$

Next, we establish the following variant of Theorem 4:

Theorem 5. Let $f$ be defined by (2-6) with $\sum_{k=1}^{n+1} \beta_{k}=1$, and suppose that

$$
\frac{\operatorname{Im}\{f(z)\}}{\operatorname{Im}\{z\}}>0 \text { for }|z| \leq 1, \operatorname{Im}\{z\} \neq 0 .
$$


Let $\theta_{ \pm}$be the interior angles of the polygon $f(\partial \mathbb{D})$ at $f( \pm 1)$. If

$$
\sum_{k=1}^{n+1}\left|\beta_{k}\right| \leq 3+\frac{1}{\pi} \max \left(\theta_{+}-\pi, 0\right)+\frac{1}{\pi} \max \left(\theta_{-}-\pi, 0\right),
$$

then $f$ is univalent.

The expression on the right-hand side of $(2-8)$ lies in $[3,5]$, and it is easy to see that any value in this range can be achieved. Therefore, Theorem 5 gives a better result than Theorem 4 under the stronger assumption (2-7). The condition (2-7) implies, in particular, that $f(\mathbb{D})$ is symmetric with respect to $\mathbb{R}$.

Proof of Theorem 5. By (2-7), the polygon $P=f(\partial \mathbb{D})$ is symmetric with respect to $\mathbb{R}$. Hence $m:=(n+1) / 2 \in \mathbb{N}$. We may assume that $z_{1}=1, z_{2}=-1$ in $(2-6)$. Then

$$
\beta_{1}=\frac{1}{2}-\frac{\theta_{+}}{2 \pi}, \quad \beta_{2}=\frac{1}{2}-\frac{\theta_{-}}{2 \pi},
$$

which satisfy $\left|\beta_{1}\right|,\left|\beta_{2}\right| \leq \frac{1}{2}$. It follows that

$$
\begin{aligned}
& \frac{1}{\pi} \max \left(\theta_{+}-\pi, 0\right)=\max \left(-2 \beta_{1}, 0\right)=\left|\beta_{1}\right|-\beta_{1}, \\
& \frac{1}{\pi} \max \left(\theta_{-}-\pi, 0\right)=\max \left(-2 \beta_{2}, 0\right)=\left|\beta_{2}\right|-\beta_{2} .
\end{aligned}
$$

Let $\varphi_{ \pm}$be the conformal mappings of $\mathbb{D}$ onto the semidiscs $\{z \in \mathbb{D}: \operatorname{Im} z \gtrless 0\}$ such that $\varphi_{ \pm}(1)=1, \varphi_{ \pm}(-1)=-1$ and $\varphi_{ \pm}( \pm i)= \pm i$. Then

$$
P_{ \pm}=f\left(\varphi_{ \pm}(\partial \mathbb{D})\right)=f(\partial \mathbb{D} \cap\{\operatorname{Im} z \gtrless 0\}) \cup[f(-1), f(+1)]
$$

are the upper and lower parts of $P$ union $[f(-1), f(1)]$. We may also assume that $\beta_{k}, k=3, \ldots, m+1$, belong to the vertices of $P$ that lie in $P_{+}$.

Consider the upper polygon $P_{+}$. The values $\gamma_{k}$ of $P_{+}$corresponding to the $\beta_{k}$ are

$$
\gamma_{k}= \begin{cases}\frac{1}{4}+\frac{1}{2} \beta_{k} \geq 0 & k=1,2, \\ \beta_{k} & k=3, \ldots, m+1,\end{cases}
$$

(for which $\sum_{k=1}^{m+1} \gamma_{k}=1$ ). In light of the symmetry with respect to $\mathbb{R}$, we get

$$
2 \sum_{k=1}^{m+1}\left|\gamma_{k}\right|=1+\beta_{1}+\beta_{2}+\sum_{k=3}^{n+1}\left|\beta_{k}\right|=1+\sum_{k=1}^{n+1}\left|\beta_{k}\right|-\left(\left|\beta_{1}\right|-\beta_{1}\right)-\left(\left|\beta_{2}\right|-\beta_{2}\right) .
$$

Using (2-8), we conclude that

$$
2 \sum_{k=1}^{m+1}\left|\gamma_{k}\right| \leq 4
$$

and it follows from Theorem 4 that $f \circ \varphi_{+}$is univalent in $\mathbb{D}$. The same holds for $f \circ \varphi_{-}$. 
By (2-9) we have

$$
f(\mathbb{D})=\left(f \circ \varphi_{+}\right)(\mathbb{D}) \cup f((-1,1)) \cup\left(f \circ \varphi_{-}\right)(\mathbb{D}),
$$

which are disjoint unions by (2-7). Hence $f$ is univalent in $\mathbb{D}$.

\section{Separation of prevertices}

Let $f$ be a Schwarz-Christoffel mapping taking $\mathbb{D}$ onto a convex $(n+1)$-gon. Recall that

$$
\frac{f^{\prime \prime}}{f^{\prime}}(z)=\frac{2 B(z)}{1-z B(z)},
$$

where $B(z)$ is a Blaschke product of degree $n$. We write

$$
B(z)=c \prod_{k=1}^{n} \frac{z-a_{k}}{1-\bar{a}_{k} z},
$$

where $|c|=1$ and all $\left|a_{k}\right|<1$. The prevertices $z_{1}, \ldots, z_{n+1}$ correspond to the roots of the equation $z B(z)=1$, and after rotating $f$, we may assume that $c=1$. Recall also that, in this case, any choice of Blaschke product $B=B(z)$ will result in a univalent mapping $f$. The separation of consecutive prevertices $z_{k}, z_{k+1}$ is to be understood as $\arg \left\{\bar{z}_{k} z_{k+1}\right\} \in(0,2 \pi)$.

Theorem 6. Suppose that $\left|a_{k}\right| \leq r<1$ for all $k$. Then:

(i) The minimum separation in argument of consecutive prevertices is given by $2 \theta$, where $\theta$ is the unique root in $(0, \pi / 2)$ of the equation

$$
\pi=\theta+2 n \arctan \left\{\frac{1+r}{1-r} \tan \frac{\theta}{2}\right\} .
$$

The result is sharp. The optimal configuration occurs when $a_{1}=\cdots=a_{n}=r c$ for some root of the equation $c^{n+1}=-1$, and the lower bound is attained for the prevertices $e^{i \theta} c, e^{-i \theta} c$. The distance between any other pair of consecutive prevertices will be larger.

(ii) The maximum separation in argument of consecutive prevertices is given by $2 \psi$, where $\psi$ is the unique root in $(0, \pi)$ of the equation

$$
\pi=\psi+2 n \arctan \left\{\frac{1-r}{1+r} \tan \frac{\psi}{2}\right\} .
$$

The result is sharp. The optimal configuration occurs when $a_{1}=\cdots=a_{n}=r d$ for some root of the equation $d^{n+1}=(-1)^{n}$, and the upper bound is attained for the prevertices $-e^{i \psi} d,-e^{-i \psi} d$. The distance between any other pair of consecutive prevertices will be larger. 
Proof. We must estimate the distance between two consecutive roots $a=e^{i \alpha}, b=e^{i \beta}$ of the equation $z B(z)=1$. Because $z B(z)$ traces the boundary $\partial \mathbb{D}$ for $z \in \partial \mathbb{D}$ in a monotonic way, we must have from (2-2) and (2-4) that

$$
\int_{\alpha}^{\beta}\left(1+\left|B^{\prime}\left(e^{i t}\right)\right|\right) d t=2 \pi,
$$

with

$$
\left|B^{\prime}\left(e^{i t}\right)\right|=\sum_{k=1}^{n} \frac{1-\left|a_{k}\right|^{2}}{\left|e^{i t}-a_{k}\right|^{2}} .
$$

(Equation (3-3) shows that $0<\beta-\alpha<2 \pi$.) We claim that for $\alpha, \beta$ fixed, the contribution of any single summand

$$
\int_{\alpha}^{\beta} \frac{1-\left|a_{k}\right|^{2}}{\left|e^{i t}-a_{k}\right|^{2}} d t
$$

will be maximal if $\left|a_{k}\right|=r$ and $a_{k} /\left|a_{k}\right|$ is the midpoint $c$ of the shorter arc joining $a$ and $b$. Let $r_{k}=\left|a_{k}\right|$ and write $a_{k}=r_{k} e^{i t_{k}}$. Then

$$
\int_{\alpha}^{\beta} \frac{1-\left|a_{k}\right|^{2}}{\left|e^{i t}-a_{k}\right|^{2}} d t=\int_{\alpha-t_{k}}^{\beta-t_{k}} \frac{1-r_{k}^{2}}{\left|1-r_{k} e^{i t}\right|^{2}} d t
$$

For $r_{k} \leq r$ given, this integral is maximal when $1 \in \partial \mathbb{D}$ is the midpoint of the shorter arc between $e^{i\left(\alpha-t_{k}\right)}$ and $e^{i\left(\beta-t_{k}\right)}$. The integral is then equal to

$$
\int_{-\theta}^{\theta} \frac{1-r_{k}^{2}}{\left|1-r_{k} e^{i t}\right|^{2}} d t=4 \arctan \left\{\frac{1+r_{k}}{1-r_{k}} \tan \frac{\theta}{2}\right\}
$$

where $2 \theta=\beta-\alpha$, and becomes maximal if $r_{k}=r$. In other words,

$$
\int_{\alpha}^{\beta} \frac{1-\left|a_{k}\right|^{2}}{\left|e^{i t}-a_{k}\right|^{2}} d t \leq 4 \arctan \left\{\frac{1+r}{1-r} \tan \frac{\theta}{2}\right\},
$$

which proves our claim for the contribution of any single term, and therefore the minimum separation between consecutive roots will occur if this holds for all $k=1, \ldots, n$. Equation (3-1) follows. The analysis shows that for the extremal configuration, all $a_{k}=r c$ are equal and that $e^{ \pm i \theta} c$ are roots of the equation $z B(z)=1$. Because $B(c)=c$, then $c B(c)=c^{n+1}$, and since $z B(z)$ traces the arc between the two roots in symmetric fashion with respect to the midpoint, we conclude that $c^{n+1}=-1$. This proves part (i).

For part (ii), we observe that, for $r_{k}=\left|a_{k}\right|$ fixed, (3-4) will be minimal provided $-1 \in \partial \mathbb{D}$ is the midpoint of the shorter arc between $e^{i\left(\alpha-t_{k}\right)}$ and $e^{i\left(\beta-t_{k}\right)}$. The integral is then equal to

$$
\int_{\pi-\psi}^{\pi+\psi} \frac{1-r_{k}^{2}}{\left|1-r_{k} e^{i t}\right|^{2}} d t=4 \arctan \left\{\frac{1-r_{k}}{1+r_{k}} \tan \frac{\psi}{2}\right\},
$$


where $2 \psi=\beta-\alpha$, and becomes minimal when $r_{k}=r$. Thus,

$$
\int_{\alpha}^{\beta} \frac{1-\left|a_{k}\right|^{2}}{\left|e^{i t}-a_{k}\right|^{2}} d t \geq 4 \arctan \left\{\frac{1-r}{1+r} \tan \frac{\theta}{2}\right\} .
$$

Therefore, the maximum separation between consecutive roots will occur if, for all $k=1, \ldots, n$, we have that $\left|a_{k}\right|=r$ and $a_{k} / r$ is equal to the midpoint of the longer arc between $a$ and $b$. From this, (3-2) follows. As before, the analysis of the extremal configuration gives $a_{k}=r d$ for all $k$. The points $-e^{ \pm i \psi} d$ are roots of $z B(z)=1$, which by symmetry as before implies that $d^{n+1}=(-1)^{n}$.

Corollary 7. Suppose that $\left|a_{k}\right| \leq \epsilon$ for all $k$. Then the maximum separation $2 \psi$ and minimum separation $2 \theta$ between consecutive prevertices satisfy

$$
\frac{\pi}{1+(1+2 \epsilon) n}+O\left(\epsilon^{2}\right) \leq \theta \leq \psi \leq \frac{\pi}{1+(1-2 \epsilon) n}+O\left(\epsilon^{2}\right), \quad \epsilon \rightarrow 0 .
$$

Proof. For fixed $x \in[0, \pi / 2]$, let $F(\delta)=\arctan ((1+\delta) \tan x)$. Then $F(0)=x$, $F^{\prime}(0)=\sin x \cos x=\frac{1}{2} \sin 2 x$ and $F^{\prime \prime}(0)=-2 \sin ^{3} x \cos x$, hence

$$
F(\delta)=x+\frac{1}{2} \sin 2 x \delta+O\left(\delta^{2}\right), \quad \delta \rightarrow 0 .
$$

Using that $(1+r) /(1-r)=1+2 r+O\left(r^{2}\right), r \rightarrow 0$, and that $\sin 2 x \leq 2 x$, we see from (3-1) that the minimum separation $\theta$ satisfies

$$
\pi \leq \theta+2 n\left(\frac{\theta}{2}+\epsilon \sin (\theta)+O\left(\epsilon^{2}\right)\right) \leq(1+(1+2 \epsilon) n) \theta+O\left(\epsilon^{2}\right) .
$$

This implies the lower bound in (3-7). A similar analysis applies to the maximum separation $\psi$, and the upper bound in (3-7) obtains.

Suppose now that $f$ is a Schwarz-Christoffel mapping taking $\mathbb{D}$ onto the complement of a bounded convex $(n+2)$-gon, with the normalization $f(0)=\infty$. In this situation, we know that

$$
z \frac{f^{\prime \prime}}{f^{\prime}}(z)=\frac{2}{z^{2} B(z)-1}
$$

where $B(z)$ again is a Blaschke product of degree $n$. We write

$$
B(z)=c \prod_{k=1}^{n} \frac{z-a_{k}}{1-\bar{a}_{k} z},
$$

where $|c|=1$ and all $\left|a_{k}\right|<1$. The prevertices $z_{1}, \ldots, z_{n+2}$ are now given by the roots of the equation $z^{2} B(z)=1$, and after a rotation of $f$, we may assume that $c=1$. The following result is obtained in a way similar to Theorem 6 , and the proof will be omitted.

Theorem 8. Suppose that $\left|a_{k}\right| \leq r<1$ for all $k$. Then: 
(i) The minimum separation in argument of consecutive prevertices is given by $2 \theta$, where $\theta$ is the unique root in $(0, \pi / 2)$ of the equation

$$
\pi=2 \theta+2 n \arctan \left\{\frac{1+r}{1-r} \tan \frac{\theta}{2}\right\} .
$$

The result is sharp. The optimal configuration occurs when $\alpha_{1}=\cdots=\alpha_{n}=r c$ for some root of the equation $c^{n+2}=-1$, and the lower bound is attained for the prevertices $e^{i \theta} c, e^{-i \theta} c$. The distance between any other pair of consecutive prevertices will be larger.

(ii) The maximum separation in argument of consecutive prevertices is given by $2 \psi$, where $\psi$ is the unique root in $(0, \pi / 2)$ of the equation

$$
\pi=2 \psi+2 n \arctan \left\{\frac{1-r}{1+r} \tan \frac{\psi}{2}\right\} \text {. }
$$

The result is sharp. The optimal configuration occurs when $\alpha_{1}=\cdots=\alpha_{n}=r d$ for some root of the equation $d^{n+2}=(-1)^{n+1}$, and the upper bound is attained for the prevertices $-e^{i \psi} d,-e^{-i \psi} d$. The distance between any other pair of consecutive prevertices will be larger.

A statement similar to Corollary 7 can be made in this case. If $\left|a_{k}\right| \leq \epsilon$ then the maximum and minimum separation between prevertices satisfy

$$
\frac{\pi}{2+(1+2 \epsilon) n}+O\left(\epsilon^{2}\right) \leq \theta \leq \psi \leq \frac{\pi}{2+(1-2 \epsilon) n}+O\left(\epsilon^{2}\right), \quad \epsilon \rightarrow 0 .
$$

We finish this section with some remarks on the separation of prevertices for arbitrary polygonal mappings. Suppose $f$ is a mapping of the form given by (1-1), where after rotation, we may assume expressions for $B_{1}, B_{2}$ given by

$$
B_{1}(z)=\prod_{k=1}^{d_{1}} \frac{z-a_{k}}{1-\bar{a}_{k} z}, \quad B_{2}(z)=\prod_{k=1}^{d_{2}} \frac{z-b_{k}}{1-\bar{b}_{k} z} .
$$

Then

$$
\varphi^{\prime}(t)=1+\left|B_{1}^{\prime}\left(e^{i t}\right)\right|-\left|B_{2}^{\prime}\left(e^{i t}\right)\right|=1+\sum_{k=1}^{d_{1}} \frac{1-\left|a_{k}\right|^{2}}{\left|e^{i t}-a_{k}\right|^{2}}-\sum_{k=1}^{d_{2}} \frac{1-\left|b_{k}\right|^{2}}{\left|e^{i t}-b_{k}\right|^{2}} .
$$

Let $a=e^{i \alpha}, b=e^{i \beta}$ be consecutive convex prevertices, with separation $\beta-\alpha=2 \delta$, and let $r$ be the radius of the smallest centered subdisk that contains the zeros of $B_{1}, B_{2}$. We deduce from (2-4) and the estimates (3-5), (3-6), that

$$
\begin{aligned}
\delta+2 d_{1} \arctan \frac{x}{\lambda}-2 d_{2} \arctan (\lambda x) & \leq \pi \\
& \leq \delta+2 d_{1} \arctan (\lambda x)-2 d_{2} \arctan \frac{x}{\lambda}
\end{aligned}
$$

where $\lambda=\frac{1+r}{1-r}$ and $x=\tan \frac{\delta}{2}$. Thus, for example, with given $d_{1}, d_{2}$, a relatively 
small separation $2 \delta$ can only occur if $r$ is rather close to 1 . Because the univalence of $f$ is no longer guaranteed when $B_{1}, B_{2}$ are chosen arbitrarily, it seems of interest to determine under which circumstances the inequalities (3-9) remain sharp. We provide here a simple example where one can show sharpness in the right-hand side of (3-9) when $d_{1}=d_{2}=1$.

Example. Consider the Blaschke products $B_{1}, B_{2}$ given by

$$
B_{1}(z)=\frac{z+r}{1+r z}, \quad B_{2}(z)=\frac{z-r}{1-r z}, \quad r \in(0,1),
$$

and let $f$ be defined, up to an affine change, by

$$
\frac{f^{\prime \prime}}{f^{\prime}}=\frac{2 B_{1} / B_{2}}{1-z B_{1} / B_{2}}=\frac{2(z+r)(1-r z)}{(z-r)(1+r z)-z(z+r)(1-r z)} \text {. }
$$

In analyzing the roots of $z B_{1}=B_{2}$, we observe that $z_{3}=1$ is one immediate solution. The other solutions are the roots of

$$
r z^{2}+\left(r^{2}+2 r-1\right) z+r=0,
$$

which are given by

$$
z_{1,2}=\frac{\left(1-2 r-r^{2}\right) \pm \sqrt{-\left(1-r^{2}\right)\left(r^{2}+4 r-1\right)}}{2 r} .
$$

For $r>r_{0}=\sqrt{5}-2=0.236 \ldots$, the discriminant is negative and $\left|z_{1,2}\right|=1$, with $z_{1}=z_{2}(=-1)$ only for $r=1$. In the partial fraction decomposition

$$
\frac{f^{\prime \prime}}{f^{\prime}}=-2\left(\frac{\beta_{1}}{z-z_{1}}+\frac{\beta_{2}}{z-z_{2}}+\frac{\beta_{3}}{z-z_{3}}\right)
$$

we must have $\beta_{1}=\beta_{2}$ because of symmetry, while $\beta_{1}+\beta_{2}+\beta_{3}=1$ by equating coefficients with the above representation for $f^{\prime \prime} / f^{\prime}$. Recall (2-3), which relates the exterior angles $2 \pi \beta_{k}$ with the boundary function $\varphi(t)$. Here

$$
\varphi^{\prime}(t)=1+\frac{1-r^{2}}{\left|1+r e^{i t}\right|^{2}}-\frac{1-r^{2}}{\left|1-r e^{i t}\right|^{2}} ;
$$

hence

$$
\varphi^{\prime}(0)=1+\frac{1-r}{1+r}-\frac{1+r}{1-r} .
$$

One readily verifies that $\varphi^{\prime}(0) \leq-2$ precisely when $r \geq r_{1}=(1+\sqrt{13}) /(5+\sqrt{13})=$ $0.535 \ldots$, in which case $\beta_{3} \in\left(-\frac{1}{2}, 0\right)$ and $\beta_{1}=\beta_{2} \in\left(\frac{1}{2}, \frac{3}{4}\right)$. Thus, for $r \geq r_{1}$, we deduce from Theorem 4 that $f$ is univalent, and $z_{1,2}$ are convex prevertices, while $z_{3}$ is a concave prevertex. The convex vertices $f\left(z_{1,2}\right)$ are at infinity, and the image $f(\mathbb{D})$ corresponds to a half-plane minus a symmetric slit ending at the concave vertex when $r=r_{1}$, or a wedge when $r>r_{1}$. 
Finally, to show sharpness in the right-hand side of (3-9), observe that for $r \geq r_{1}$ the conjugate points $z_{1}=\bar{z}_{2}$ have negative real part, and thus their separation $2 \theta$ will correspond to the root $\theta \in(0, \pi / 2)$ of the equation

$$
\theta+2 \arctan (\lambda x)-2 \arctan \frac{x}{\lambda}=\pi .
$$

For consecutive concave prevertices, we deduce in similar fashion that

$$
\begin{aligned}
\delta+2 d_{1} \arctan \frac{x}{\lambda}-2 d_{2} \arctan (\lambda x) & \leq-\pi \\
& \leq \delta+2 d_{1} \arctan (\lambda x)-2 d_{2} \arctan \frac{x}{\lambda},
\end{aligned}
$$

once again forcing $r$ to be very close to 1 if a small separation is to happen.

A similar analysis can be carried through to obtain information about the separation between consecutive convex or concave prevertices in the case of exterior mappings. The resulting inequalities are analogous to (3-9) and (3-10), with the single term $\delta$ replaced by $2 \delta$. The proof will be omitted.

\section{Location of zeros}

In this section we study the location of the zeros of the Blaschke products appearing in the representation formulas (1-1) and (1-4) of Schwarz-Christoffel mappings. Convex or concave mappings impose no restriction on the location of the zeros, since in the absence of the Blaschke product $B_{2},(1-1)$ and (1-4) will always render univalent mappings. It is probably an ambitious task to determine conditions on $B_{1}$ and $B_{2}$ that are both necessary and sufficient for all mappings of the form (1-1) and (1-4) to be univalent. Nevertheless, some necessary conditions can be established. We deal first with the case of mappings arising from (1-1). Because $1+\operatorname{Re}\left\{z f^{\prime \prime} / f^{\prime}\right\}$ will be positive or negative according to whether $\left|z B_{1}\right|<\left|B_{2}\right|$ or $\left|z B_{1}\right|>\left|B_{2}\right|$, it follows readily from the radius of convexity for the class $\mathscr{S}$ that we must have

$$
\left|z B_{1}(z)\right|<\left|B_{2}(z)\right|, \quad|z| \leq 2-\sqrt{3} .
$$

In particular, all zeros of $B_{2}$ must lie in the region $|z|>2-\sqrt{3}$.

Theorem 9. Let $f$ be given by (1-1), with $d_{1}, d_{2}$ the degrees of the Blaschke products $B_{1}, B_{2}$, respectively, and suppose that $d_{2} \geq 1$. Suppose that all the zeros of $B_{1}, B_{2}$ are contained in the subdisk $|z| \leq r<1$. Then

$$
r \geq \max \left\{\frac{\sqrt{4 d_{1} d_{2}+9}+3-2 d_{2}}{\sqrt{4 d_{1} d_{2}+9}+3+2 d_{2}}, \frac{2 d_{2}-1-\sqrt{1+4 d_{1} d_{2}}}{2 d_{2}+1+\sqrt{1+4 d_{1} d_{2}}}\right\} .
$$

In particular, if $d_{2}=1$ then

$$
r \geq \frac{\sqrt{4 n+5}+1}{\sqrt{4 n+5}+5} \geq \frac{1}{2}
$$


The estimate (4-2) is sharp for the Koebe function.

Proof. Recall the boundary function $\varphi(t)$ in (2-2). At a concave prevertex $e^{i t_{0}}$, the exterior angle $2 \pi \beta_{0}$ lies in $[-\pi, 0)$, and hence $\beta_{0}$ lies in $\left[-\frac{1}{2}, 0\right)$. It follows from (2-3) that

$$
\varphi^{\prime}\left(t_{0}\right) \leq-2
$$

If we write

$$
B_{1}(z)=c_{1} \prod_{k=1}^{d_{1}} \frac{z-a_{k}}{1-\bar{a}_{k} z}, \quad B_{2}(z)=c_{2} \prod_{k=1}^{d_{2}} \frac{z-b_{k}}{1-\bar{b}_{k} z}
$$

then

$$
\varphi^{\prime}(t)=1+\sum_{k=1}^{d_{1}} \frac{1-\left|a_{k}\right|^{2}}{\left|e^{i t}-a_{k}\right|^{2}}-\sum_{k=1}^{d_{2}} \frac{1-\left|b_{k}\right|^{2}}{\left|e^{i t}-b_{k}\right|^{2}} .
$$

After evaluating at $t=t_{0}$, a simple estimate gives

$$
1+d_{1} \frac{1-r}{1+r}-d_{2} \frac{1+r}{1-r} \leq-2 .
$$

With $s=(1+r) /(1-r)$, we obtain

$$
d_{2} s^{2}-3 s-d_{1} \geq 0
$$

which implies (4-1). If $d_{2}=1$, then $d_{1}=n-1$, which proves the first estimate in (4-2).

In order to obtain the second estimate, we observe that at a convex prevertex $e^{i t_{1}}$, the exterior angle $2 \pi \beta_{1}$ is positive, and therefore $\varphi^{\prime}\left(t_{1}\right)>0$. This now gives

$$
1+d_{1} \frac{1+r}{1-r}-d_{2} \frac{1-r}{1+r}>0,
$$

and the second estimate follows.

To show sharpness, we consider the Koebe function $k(z)=z /(1-z)^{2}$. Then $k^{\prime}(z)=(1+z) /(1-z)^{3}$, and thus

$$
\frac{k^{\prime \prime}}{k^{\prime}}(z)=\frac{1}{z+1}-\frac{3}{z-1},
$$

which is consistent with a polygonal mapping onto a 2-gon with a concave vertex with exterior angle $-\pi$ at $k(-1)=-\frac{1}{4}$, and a convex vertex with exterior angle $3 \pi$ at $k(1)=\infty$. A calculation gives

$$
\frac{k^{\prime \prime}}{k^{\prime}}(z)=\frac{1 / B_{2}(z)}{1-z / B_{2}(z)},
$$

with $B_{2}(z)=\left(z+\frac{1}{2}\right) /\left(1+\frac{1}{2} z\right)$. Then $r=\frac{1}{2}$, which coincides with the lower bound in (4-2) with $n=1$. 
Remark. The first estimate in (4-1) is the better one when $d_{1} \gg d_{2}$, while the second will provide better information for $d_{2} \gg d_{1}$.

The final theorem describes the analogous situation for mappings of the form (1-4) onto the complement of polygons. The corresponding boundary function is now given by

$$
\varphi(t)=\arg \left\{e^{2 i t} \frac{B_{1}}{B_{2}}\left(e^{i t}\right)\right\}
$$

for which

$$
\varphi^{\prime}(t)=2+\left|B_{1}^{\prime}\left(e^{i t}\right)\right|-\left|B_{2}^{\prime}\left(e^{i t}\right)\right| .
$$

Since the proof is based on an almost identical analysis, it will be omitted.

Theorem 10. Let $f$ be given by (1-4), with $d_{1}, d_{2}$ the degrees of the Blaschke products $B_{1}, B_{2}$, respectively, and suppose that $d_{2} \geq 1$. Suppose that all the zeros of $B_{1}, B_{2}$ are contained in the subdisk $|z| \leq r<1$. Then

$$
r \geq \max \left\{\frac{\sqrt{d_{1} d_{2}+4}+2-d_{2}}{\sqrt{d_{1} d_{2}+4}+2+d_{2}}, \frac{d_{2}-1-\sqrt{1+d_{1} d_{2}}}{d_{2}+1+\sqrt{1+d_{1} d_{2}}}\right\} .
$$

\section{Acknowledgement}

We thank the referee for a very careful reading of the manuscript and several valuable suggestions.

\section{References}

[Avkhadiev and Wirths 2002] F. G. Avkhadiev and K.-J. Wirths, "Convex holes produce lower bounds for coefficients", Complex Var. Theory Appl. 47:7 (2002), 553-563. MR 2003f:30013 Zbl 1028.30010

[Avkhadiev and Wirths 2005] F. G. Avkhadiev and K.-J. Wirths, "Concave schlicht functions with bounded opening angle at infinity”, Lobachevskii J. Math. 17 (2005), 3-10. MR 2005m:30013 Zbl 1071.30005

[Avkhadiev and Wirths 2007] F. G. Avkhadiev and K.-J. Wirths, "A proof of the Livingston conjecture", Forum Math. 19:1 (2007), 149-157. MR 2007k:30027 Zbl 1109.30021

[Bhowmik et al. 2009] B. Bhowmik, S. Ponnusamy, and K.-J. Wirths, "Вогнутые функции, произведения Бляшке и полигональные отображения”, Sibirsk. Mat. Zh. 50:4 (2009), 772-779. Translated as "Concave functions, Blaschke products, and polygonal mappings" in Sib. Math. J. 50:4 (2009), 609-615. MR 2011a:30123 Zbl 1224.30035

[Chuaqui et al. 2011] M. Chuaqui, P. L. Duren, and B. Osgood, "Schwarzian derivatives of convex mappings", Ann. Acad. Sci. Fenn. Math. 36:2 (2011), 449-460. MR 2012j:30024 Zbl 1239.30003

[Chuaqui et al. 2012] M. Chuaqui, P. L. Duren, and B. Osgood, "Concave conformal mappings and pre-vertices of Schwarz-Christoffel mappings", Proc. Amer. Math. Soc. 140:10 (2012), 3495-3505. MR 2929018 Zbl 1283.30048

[Duren 1983] P. L. Duren, Univalent functions, Grundlehren der Mathematischen Wissenschaften 259, Springer, New York, 1983. MR 85j:30034 Zbl 0514.30001 
Received May 13, 2013. Revised November 29, 2013.

MARTIN CHUAQUi

FACUltad de Matemáticas

Pontificia Universidad Católica de ChILE

CASILla 306

22 SANTIAGO

CHILE

mchuaqui@mat.puc.cl

Christian POMMERENKe

INSTITUT FÜR MATHEMATIK MA 8-1

TECHNISCHE UNIVERSITÄT

D-10623 BERLIN

GERMANY

pommeren@math.tu-berlin.de 


\title{
PACIFIC JOURNAL OF MATHEMATICS
}

\author{
msp.org/pjm
}

Founded in 1951 by E. F. Beckenbach (1906-1982) and F. Wolf (1904-1989)

\section{EDITORS}

Don Blasius (Managing Editor)

Department of Mathematics

University of California

Los Angeles, CA 90095-1555

blasius@math.ucla.edu

\author{
Paul Balmer \\ Department of Mathematics \\ University of California \\ Los Angeles, CA 90095-1555 \\ balmer@math.ucla.edu \\ Robert Finn \\ Department of Mathematics \\ Stanford University \\ Stanford, CA 94305-2125 \\ finn@math.stanford.edu \\ Sorin Popa \\ Department of Mathematics \\ University of California \\ Los Angeles, CA 90095-1555 \\ popa@math.ucla.edu
}

\author{
Vyjayanthi Chari \\ Department of Mathematics \\ University of California \\ Riverside, CA 92521-0135 \\ chari@math.ucr.edu \\ Kefeng Liu \\ Department of Mathematics \\ University of California \\ Los Angeles, CA 90095-1555 \\ liu@math.ucla.edu \\ Jie Qing \\ Department of Mathematics \\ University of California \\ Santa Cruz, CA 95064 \\ qing@ cats.ucsc.edu
}

\section{PRODUCTION}

Silvio Levy, Scientific Editor, production@msp.org

\section{SUPPORTING INSTITUTIONS}

ACADEMIA SINICA, TAIPEI

CALIFORNIA INST. OF TECHNOLOGY

INST. DE MATEMÁTICA PURA E APLICADA

KEIO UNIVERSITY

MATH. SCIENCES RESEARCH INSTITUTE

NEW MEXICO STATE UNIV.

OREGON STATE UNIV.

\author{
STANFORD UNIVERSITY \\ UNIV. OF BRITISH COLUMBIA \\ UNIV. OF CALIFORNIA, BERKELEY \\ UNIV. OF CALIFORNIA, DAVIS \\ UNIV. OF CALIFORNIA, LOS ANGELES \\ UNIV. OF CALIFORNIA, RIVERSIDE \\ UNIV. OF CALIFORNIA, SAN DIEGO \\ UNIV. OF CALIF., SANTA BARBARA
}

\author{
Daryl Cooper \\ Department of Mathematics \\ University of California \\ Santa Barbara, CA 93106-3080 \\ cooper@math.ucsb.edu \\ Jiang-Hua Lu \\ Department of Mathematics \\ The University of Hong Kong \\ Pokfulam Rd., Hong Kong \\ jhlu@maths.hku.hk \\ Paul Yang \\ Department of Mathematics \\ Princeton University \\ Princeton NJ 08544-1000 \\ yang@math.princeton.edu
}

These supporting institutions contribute to the cost of publication of this Journal, but they are not owners or publishers and have no responsibility for its contents or policies.

See inside back cover or msp.org/pjm for submission instructions.

The subscription price for 2014 is US $\$ 410 /$ year for the electronic version, and \$535/year for print and electronic.

Subscriptions, requests for back issues and changes of subscribers address should be sent to Pacific Journal of Mathematics, P.O. Box 4163, Berkeley, CA 94704-0163, U.S.A. The Pacific Journal of Mathematics is indexed by Mathematical Reviews, Zentralblatt MATH, PASCAL CNRS Index, Referativnyi Zhurnal, Current Mathematical Publications and Web of Knowledge (Science Citation Index).

The Pacific Journal of Mathematics (ISSN 0030-8730) at the University of California, c/o Department of Mathematics, 798 Evans Hall \#3840, Berkeley, CA 94720-3840, is published twelve times a year. Periodical rate postage paid at Berkeley, CA 94704, and additional mailing offices. POSTMASTER: send address changes to Pacific Journal of Mathematics, P.O. Box 4163, Berkeley, CA 94704-0163.

PJM peer review and production are managed by EditFLOW ${ }^{\circledR}$ from Mathematical Sciences Publishers.

\section{PUBLISHED BY}

\section{mathematical sciences publishers \\ nonprofit scientific publishing}

http://msp.org/

(C) 2014 Mathematical Sciences Publishers 


\section{PACIFIC JOURNAL OF MATHEMATICS}

Volume $270 \quad$ No. $2 \quad$ August 2014

Disjointification inequalities in symmetric quasi-Banach spaces and 257 their applications

SERgey Astashinin, Fedor A. SukocheV and DMitriy

ZANIN

Hamiltonian evolutions of twisted polygons in parabolic manifolds:

287

The Lagrangian Grassmannian

GLORIA MARÍ BEFFA

On Schwarz-Christoffel mappings

MARTIN CHUAQUi and CHRISTIAN POMMERENKE

Vanishing viscosity in the plane for nondecaying velocity and vorticity, II

ELAINE COZZI

Affine quantum Schur algebras and affine Hecke algebras

QIANG FU

On the classification of Killing submersions and their isometries

JosÉ M. MANZANO

Locally Lipschitz contractibility of Alexandrov spaces and its applications

Ayato Mitsuishi and TAKaO Yamaguchi

Sequences of open Riemannian manifolds with boundary

RAQUEL PERALES and CHRISTINA SORMANI

Invariant differential operators on a class of multiplicity-free spaces 